\title{
FORMULÁRIO DE SEGUIMENTO FARMACOTERAPÊUTICO A CUIDADORES DE PACIENTES COM ESQUIZOFRENIA REFRATÁRIA
}

\section{ARTIGO DE REVISÃO}

SILVA, Aglaciene Lopes da ${ }^{1}$

BASTOS, Camila Granado de ${ }^{2}$

DOMINGOS, Flavia Caires ${ }^{3}$

PINTO, Michele Cristina da Rocha ${ }^{4}$

SANTANA, Claudinei Alves ${ }^{5}$

SILVA, Aglaciene Lopes da. Et al. Formulário de seguimento farmacoterapêutico a cuidadores de pacientes com esquizofrenia refratária. Revista Científica Multidisciplinar Núcleo do Conhecimento. Ano 05, Ed. 09, Vol. 05, pp. 93-107. Setembro de 2020. ISSN: 2448-0959, Link de acesso: https://www.nucleodoconhecimento.com.br/saude/esquizofrenia-refrataria

\section{RESUMO}

Introdução: A esquizofrenia é um dos transtornos mentais de maior relevância na saúde pública, ocasionando grande aflição para o doente e seus familiares. A persistência dos sintomas caracteriza refratariedade da doença que é tratada com a clozapina considerada padrão-ouro no tratamento, mas suas reações adversas

\footnotetext{
${ }^{1}$ Farmacêutica. Especialista em Farmacologia Clínica e Farmacoterapia, Senac.

${ }^{2}$ Farmacêutica. Especialista em Farmacologia Clínica e Farmacoterapia, Senac.

${ }^{3}$ Farmacêutica. Especialista em Farmacologia Clínica e Farmacoterapia, Senac.

${ }^{4}$ Farmacêutica. Especialista em Farmacologia Clínica e Farmacoterapia, Senac.

${ }^{5}$ Farmacêutico. Mestre em Ciências Médicas, FMUSP. Especialista em Oncologia Multiprofissional (HSL). Especialista em Farmácia Hospitalar (FOC).
} 
podem ser tão intensas quanto os sintomas do transtorno. Objetivo: Desenvolver um formulário de seguimento farmacoterapêutico a cuidadores de pacientes adultos com esquizofrenia refrataria a partir da utilização da clozapina. Métodos: A busca pelos artigos foi realizada nas bases de dados e sites governamentais no período de 15 de novembro de 2018 a 15 de janeiro de 2019, publicados a partir de 2012. Resultado: Foram encontrados 37 artigos, excluídos 20 artigos, incluindo nesta revisão 17 artigos, 2 livros, 4 protocolos clínicos e 1 monografia do fármaco. Conclusão: A proposta de elaboração do formulário de seguimento farmacoterapêutico é identificar as reações adversas que possam influenciar a adesão ao tratamento farmacológico e reduzir os efeitos indesejados do tratamento como consequência melhorar a qualidade de vida paciente.

Palavras-chave: atenção farmacêutica, esquizofrenia, clozapina, cuidadores.

\section{INTRODUÇÃO}

Esquizofrenia ou transtornos esquizofrênicos constituem um grupo de distúrbios mentais graves, sem sintomas patognomônicos, caracterizados por distorções do pensamento e da percepção, por inadequação e dificuldade de expressar afeto sem prejuízo da capacidade intelectual, apresentando ao longo do tempo prejuízos cognitivos (PEREIRA et al., 2013)

São caracterizadas pela presença de sintomas positivos (delírios, alucinações e desorganização do pensamento e da conduta, ideias bizarras, agitação psicomotora e alterações de linguagem) e sintomas negativos (embotamento afetivo-volitivo), perdas cognitivas (sobretudo déficit da capacidade de abstração e insight) e sintomas depressivos e ansiosos. (CEZARETTO et al., 2014)

O primeiro episódio apresenta-se na forma de um surto psicótico, por volta dos 20 anos, nos homens e 25 nas mulheres, normalmente associado á alucinações, delírios e desorganização do pensamento durante as crises agudas; intercalados por períodos de remissão, com dificuldade de expressão das emoções, apatia, isolamento social e um sentimento profundo de desesperança. (SILVA et al., 2016) 
A esquizofrenia é um dos transtornos mentais de maior relevância na saúde pública, ocasionando grande aflição para o doente e seus familiares. (MAGALHÃES et al., 2018)

Cerca de $30 \%$ das pessoas que possuem esquizofrenia então apresentam melhora do quadro clínico, sendo denominadas refratárias ao tratamento, sendo que a principal característica da Esquizofrenia Refratária é a persistência dos sintomas positivos de moderada a grave intensidade, apesar do tratamento adequado (PEREIRA et al., 2013; CEZARETTO et al., 2014). Segundo a Organização Mundial de Saúde (OMS) em 2018 estimou-se que existia 21 milhões de pessoas com esquizofrenia no mundo, causando importante impacto cognitivo, funcional e afetivo (PEREIRA et al., 2013; WHO, 2019).

No Brasil em 2008 estimou-se cerca de 1,6 milhões de pacientes esquizofrênicos, impactando o sistema de saúde demandando assistência especifica. (Oliveira et al., 2017)

O tratamento da esquizofrenia é composto pela terapêutica medicamentosa (antipisicóticos), psicoterapia e socioterapia. (FREITAS et al., 2016)

Os fármacos antipsicóticos são antagonistas ou agonistas parciais nos receptores D2 de dopamina, porém a maioria também bloqueia vários outros receptores de monoaminas. A potência dos antipisicóticos, em geral, corre paralela a atividade dos receptores D2, mas atividades em outros receptores (5-HT2A, e muscarínicos), podem reduzir os efeitos extrapiramidais adversos. (RANG et al., 2007)

Os antipsicóticos podem ainda ser divididos em antipsicóticos típicos (haloperidol, flufenazina, primozida, clorpromazina, levomepromazina) isto é, fármacos mais antigos com ações proeminentes no receptor D2, e antipsicóticos atípicos (clozapina, olanzapina, quetiapina, ziprasidona e risperidona, que constituem uma geração mais nova de fármacos com antagonismo D2 menos proeminente e consequentemente com menos efeitos extrapiramidais. (GOLAN, 2009) 
Os tratamentos devem ser feitos em monoterapia de acordo com o perfil de segurança e a tolerabilidade do paciente (CEZARETTO et al., 2014). Em caso de falha terapêutica com o uso de qualquer desses fármacos por pelo menos 6 semanas nas doses adequadas sem melhora de pelo menos 30\% na escala de Avaliação Psiquiátrica, uma segunda tentativa com algum outro antipsicótico deverá ser feita (BRASIL, 2013).

Para pacientes que possui a forma mais grave de esquizofrenia, chamada de refratária ou resistente, o tratamento de escolha é o uso do antipsicótico atípico, a clozapina, considerada padrão-ouro no que demonstra reduzir os sintomas agudos e o risco de suicídio (FREITAS et al., 2016; CAMPANA E SOARES, 2015). Não tendo evidências de que a associação com outro antipsicótico possa trazer benefícios ao paciente (PEREIRA et al., 2013).

Todos os antipsicóticos, com exceção de clozapina, podem ser utilizados no tratamento sem ordem de preferência em pacientes com diagnóstico de Esquizofrenia (BRASIL, 2013).

Para o controle da esquizofrenia o tratamento medicamentoso é fundamental, mas na avaliação dos pacientes, as reações adversas acarretadas pelo tratamento medicamentoso podem ser tão intensas quanto os sintomas do transtorno, sendo elas alterações motoras extrapiramidais, alterações endócrinas, sedação, hipotensão e ganho de peso, boca seca, visão embaçada, agranulocitose (SILVA et al., 2016; GOLAN et al., 2009).

A Organização Mundial de Saúde define reação adversa a medicamento (RAM) como "qualquer resposta prejudicial ou indesejável e não intencional que ocorre com medicamentos em doses normalmente utilizadas no homem para profilaxia, diagnóstico, tratamento de doença ou para modificação de funções fisiológicas" (WHO, 2019).

A família passa a ser protagonista nos cuidados de pessoas portadoras de esquizofrenia sendo importante para sua ressocialização e, pois, é a primeira e 
principal instituição social com que o portador de transtorno mental vai se socializar (BENNETT et al., 2015; CAMPANA E SOARES, 2015). É importante que seus cuidadores e familiares estejam preparados para conviverem com a dor e sofrimento do doente (CEZARETTO et al., 2014; MAGALHAES et al., 2018).

Uma das principais preocupações do cuidado na saúde mental é a polifarmácia com uso exagerado e inadequado de medicamentos, que poderá ser adaptado pela assistência, atenção ou atividade clínica do farmacêutico (FEGADOLLI et al., 2016). A participação do farmacêutico vem sendo fundamental, podendo estabelecer o primeiro contato com o paciente e atuar como um elo com os demais profissionais da equipe de saúde mental participando de estratégias de adesão e reações adversas atuando no cuidado à saúde mental (MAGALHAES et al., 2018; FEGADOLLI et al., 2016; SILVA E LIMA, 2017).

\section{OBJETIVO}

Desenvolver um formulário de seguimento farmacoterapêutico a cuidadores de pacientes adultos com esquizofrenia refrataria a partir da utilização da clozapina.

\section{METODOLOGIA}

A busca pelos artigos foi realizada nas bases de dados: Scielo (Scientific Electronic Library Online) e PubMed (National Library of Medicine), NCBI (National Center for Biotechnology Information), BVS (Biblioteca Virtual em Saúde), sites governamentais (Ministério da Saúde, Agencia Nacional de Vigilância Sanitária, Organização Mundial de Saúde) em língua portuguesa e inglesa. A busca foi realizada de 15 de novembro de 2018 a 15 de janeiro de 2019.

As palavras chaves utilizadas em português foram atenção farmacêutica, esquizofrenia, clozapina e cuidadores e em inglês pharmaceutical attention, schizophrenia, clozapine e caregivers.

Foram considerados validos artigos publicados no período de 2013 a 2018 com acesso livre e texto completo. 


\section{RESULTADOS}

A partir da metodologia adotada foram encontrados 37 artigos que após a leitura exaustiva foram excluídos 20 artigos, pois, não demonstravam clareza na temática proposta.

Sendo assim, foram incluídos nesta revisão 17 artigos, 2 livros, 4 protocolos clínicos e 1 monografia do fármaco.

\section{DISCUSSÃO}

As reações adversas a medicamentos (RAM) são mais comuns do que se pode esperar e nunca se pode garantir que um medicamento seja completamente seguro. A utilização de medicamentos em situações não indicadas ou em circunstâncias que desrespeitem os critérios de uso racional podem provocar danos, aumentar a frequência de internações hospitalares, custos a saúde e até mesmo o óbito.

A clozapina é o tratamento de escolha na esquizofrenia refratária e o seu efeito terapêutico promove um aprimoramento do raciocínio lógico, situação essencial para a compreensão da doença e adesão ao tratamento, acarretando assim uma importante melhoria clínica dos pacientes e, consequentemente, de sua qualidade de vida (BARKHOF et al., 2012; BATISTA et al., 2016; SISKIND et al., 2016).

Todavia, o uso da clozapina não está isento de efeitos adversos, alguns dos quais potencialmente ameaçam a vida, são limitações importantes para não aderência (PORRAS-SEGOVIA et al., 2017). Preocupações com outros efeitos adversos que incluem, falta de apetite, sedação, ganho de peso, resistência à insulina, que são relacionados ao desenvolvimento da síndrome metabólica $(\mathrm{SM})$ e forte atividade anticolinérgica intrínseca podem aumentar a resistência para tentar esta medicação (BATISTA et al., 2016; SISKIND et al., 2016; PORRAS-SEGOVIA et al., 2017).

Deve-se considerar a presença de um familiar ou um indivíduo responsável por cuidar do paciente, ajudando na execução das atividades diárias, já que a gravidade da 
doença gera estresse, isolamento e custos financeiros adicionais, tornando os cuidadores sobrecarregados (KHAN E ZAIDI, 2017; HANSEN et al., 2014).

Em relação à clozapina uma reação adversa de importante impacto no paciente é a agranulocitose, caracterizada pela diminuição das células brancas granulocíticas sendo uma das principais razões para a descontinuação do tratamento (SISKIND et al., 2016; KHAN E ZAIDI, 2017). Esse risco para agranulocitose, tem uma prevalência de 1-2\% nos pacientes tratados com clozapina, situação monitorada obrigatoriamente pelos profissionais da saúde para assegurar que os pacientes recebam os medicamentos rotineiramente (WARNEZ E ALESSI-SEVERINI, 2014). As atuais monografias de produtos determinam que os pacientes iniciem a terapia gradualmente e que as contagens de leucócitos e neutrófilos (CAN) sejam mantidas em níveis seguros (CRISTALIA, 2019).

Para o acompanhamento do paciente e seu cuidador foi elaborado um formulário de seguimento farmacoterapêutico, de preenchimento mensal, com as RAMs muito comuns e comuns da clozapina, selecionadas inicialmente por serem reações que estão em mais evidências nos estudos clínicos, serem mais fáceis de identificar, e por serem motivos de maior desistência do tratamento. Outras RAMs do fármaco não foram utilizadas por necessitarem de uma atenção médica especializada e de resultados de exames laboratoriais.

A partir da revisão bibliográfica foram selecionados os campos obrigatórios presentes no formulário de seguimento farmacoterapêutico apresentados abaixo:

- Identificação do paciente: Neste bloco identificam-se as características do paciente quanto ao nome, sexo, data de nascimento, idade medidas antropométricas (altura e peso). A avaliação do peso é importante, pois uma das reações adversas é o ganho de peso que esta relacionada com a Síndrome Metabólica (SM) (BATISTA, 2016). Existem evidências de que pessoas com esquizofrenia refratária que utilizam clozapina estão expostas a um risco mais elevado para SM, em comparação à esquizofrenia de modo geral, podendo 
alcançar o dobro do peso, representando um grave problema de saúde pública a nível mundial (BATISTA, 2016).

- Condição clínica do paciente: Neste bloco identificam-se as limitações do paciente quanto à visão, fala, locomoção e audição. Havendo alguma limitação será necessário a presença de um cuidador.

- Hábitos de vida e vícios: Neste bloco identifica-se o consumo de álcool, uso de cigarro e drogas ilícitas: Essa informação é importante, pois a clozapina potencializa os efeitos do álcool e outros depressores do sistema nervoso central, e se o paciente for tabagista a interrupção súbita do tabagismo aumenta a concentração plasmática da clozapina, levando a um aumento das reações adversas (CRISTALIA, 2019)

- Hábitos alimentares e atividade física: Neste bloco identifica-se a frequência diária da alimentação e prática de atividade física com o intuito de intervenção para melhorar da qualidade de vida do paciente uma vez que a ingestão de cafeína, pode aumentar a concentração plasmática da clozapina (CRISTALIA, 2019).

- Histórico de uso medicamento e alergias: Neste bloco identifica-se a história de uso e alergias a outros medicamentos a fim de minimizar os efeitos maléficos interações medicamentosas.

- Tratamento medicamentoso com clozapina: Neste bloco identifica-se a doses diárias e horários de administração, a fim de minimizar os efeitos maléficos das interações medicamentosas.

- Avaliação da adesão ao tratamento: Neste bloco busca identificar se o paciente apresenta dificultadores da adesão ao tratamento com intuito de orientá-lo quanto ao uso correto para um maior efeito terapêutico.

- Avaliação das Reações Adversas ao Medicamento (RAM's): Neste bloco o paciente e/ou cuidador indicará a presença de alguma reação adversa ao medicamento descrevendo a sua frequência. 
Tabela 1. Reações Adversas ao Medicamento Comuns e Muito Comuns separadas por sistemas fisiológicos (CRISTALIA, 2019).

\begin{tabular}{|c|c|c|}
\hline $\begin{array}{l}\text { Distúrbio por sistema } \\
\text { fisiológico }\end{array}$ & RAM comuns & RAM muito comuns \\
\hline $\begin{array}{l}\text { Distúrbios metabólicos e } \\
\text { nutricionais }\end{array}$ & Ganho de peso & \\
\hline Distúrbios psiquiátricos & $\begin{array}{l}\text { Disartria (fraqueza } \\
\text { muscular, dificuldade ao } \\
\text { falar) }\end{array}$ & \\
\hline $\begin{array}{l}\text { Distúrbios do sistema } \\
\text { nervoso }\end{array}$ & $\begin{array}{l}\text { Convulsões, abalos } \\
\text { mioclônicos, sintomas } \\
\text { extrapiramidais, tremores, } \\
\text { rigidez, dor de cabeça }\end{array}$ & $\begin{array}{l}\text { Sonolência, } \\
\text { vertigem }\end{array}$ \\
\hline Distúrbios visuais & Visão borrada & \\
\hline Distúrbios cardíacos & $\begin{array}{l}\text { Alteração no ECG } \\
\text { (eletrocardiograma) }\end{array}$ & Taquicardíaca \\
\hline $\begin{array}{l}\text { Distúrbios do sistema } \\
\text { vascular }\end{array}$ & $\begin{array}{l}\text { Hipotensão postural, } \\
\text { hipertensão, sincope }\end{array}$ & \\
\hline Distúrbios gastrintestinais & Náusea, vômito, boca seca & $\begin{array}{l}\text { Constipação, } \\
\text { hipersalivação }\end{array}$ \\
\hline
\end{tabular}




\begin{tabular}{|l|l|}
\hline Distúrbios hepatobiliares & $\begin{array}{l}\text { Enzimas } \\
\text { elevadas }\end{array}$ \\
\hline $\begin{array}{l}\text { Distúrbios renais e eáticas } \\
\text { urinários }\end{array}$ & $\begin{array}{l}\text { Retenção urinária, } \\
\text { incontinência urinária }\end{array}$ \\
\hline Distúrbios gerais & $\begin{array}{l}\text { Febre (hipermia benigna), } \\
\text { distúrbios na sudorese, }\end{array}$ \\
& fadiga \\
\hline
\end{tabular}

Fonte: Elaboração própria

- Identificação do responsável pelo preenchimento: próprio paciente ou cuidador.

- Conduta Farmacêutica: Neste campo será determinada a conduta e orientações farmacoterapêuticas a serem transmitidas ao paciente ou cuidador para melhoraria da qualidade de vida do paciente.

Figura 1. Ficha de Acompanhamento Farmacoterapêutico de Pacientes em uso de Clozapina (BRASIL, 2019; CFF, 2019)

Ficha de Acompanhamento Farmacoterapêutico de Pacientes em uso de Clozapina

Nome

Paciente:

Sexo:

Data Nasc: 1

Altura:

Tem alguma limitação（） Sim, qual? （） Não

Visão（） Fala（） Locomoção（） Audição

( ) 
Tabagista ( ) SiM ( ) Não Quant.cigarro/dia

Faz uso de Álcool（） Sim （）Não

frequência? vezes por semana

Faz uso drogas ( ) Sim, qual?

Não

Descrever

Hábitos Alimentares: Quais refeições faz ao dia/ horário?

Café da Lanche
manhã da manhã Almoço $\begin{aligned} & \begin{array}{l}\text { Lanche } \\ \text { da tarde }\end{array} \\ & \text { Jantar }\end{aligned} \begin{aligned} & \text { Ceia da } \\ & \text { noite }\end{aligned}$

Tipos

de

Alimentos:

$\begin{array}{lcccc}\text { Pratica } & \text { Exercício } & \text { Físico } & ( & \text { Sim, } \\ \text { qual? } & (\text { )Não } & \text { Descrever } & \end{array}$

\begin{tabular}{|l|l|l|}
\hline $\begin{array}{c}\text { Faz uso de outros medicamentos } \\
\text { Medicamento }\end{array}$ & Dose diária & Horário \\
\hline & & \\
\hline & & \\
\hline & & \\
\hline
\end{tabular}


Tem alergia a algum medicamento

(

)

Sim,

qual?

( ) Não

Tratamento Atual

Medicamento

Dose diária

Horário

\section{Clozapina}

Avaliação de Adesão ao Tratamento

Tem dificuldade em tomar o comprimido ( ) Sim, qual ( ) Não

Descrever

Já se esqueceu de tomar o comprimido? （） Sim （） Não

O medicamento é tomado no horário indicado ?( ) Sim （）Não

Quando sente algum desconforto deixa de tomar o medicamento ? ( ) Sim （ ) Não

Quando se sente bem deixa de tomar o medicamento? ( ) Sim （ ) Não

Avaliação das Reações Adversas ao Medicamento(RAM's) 


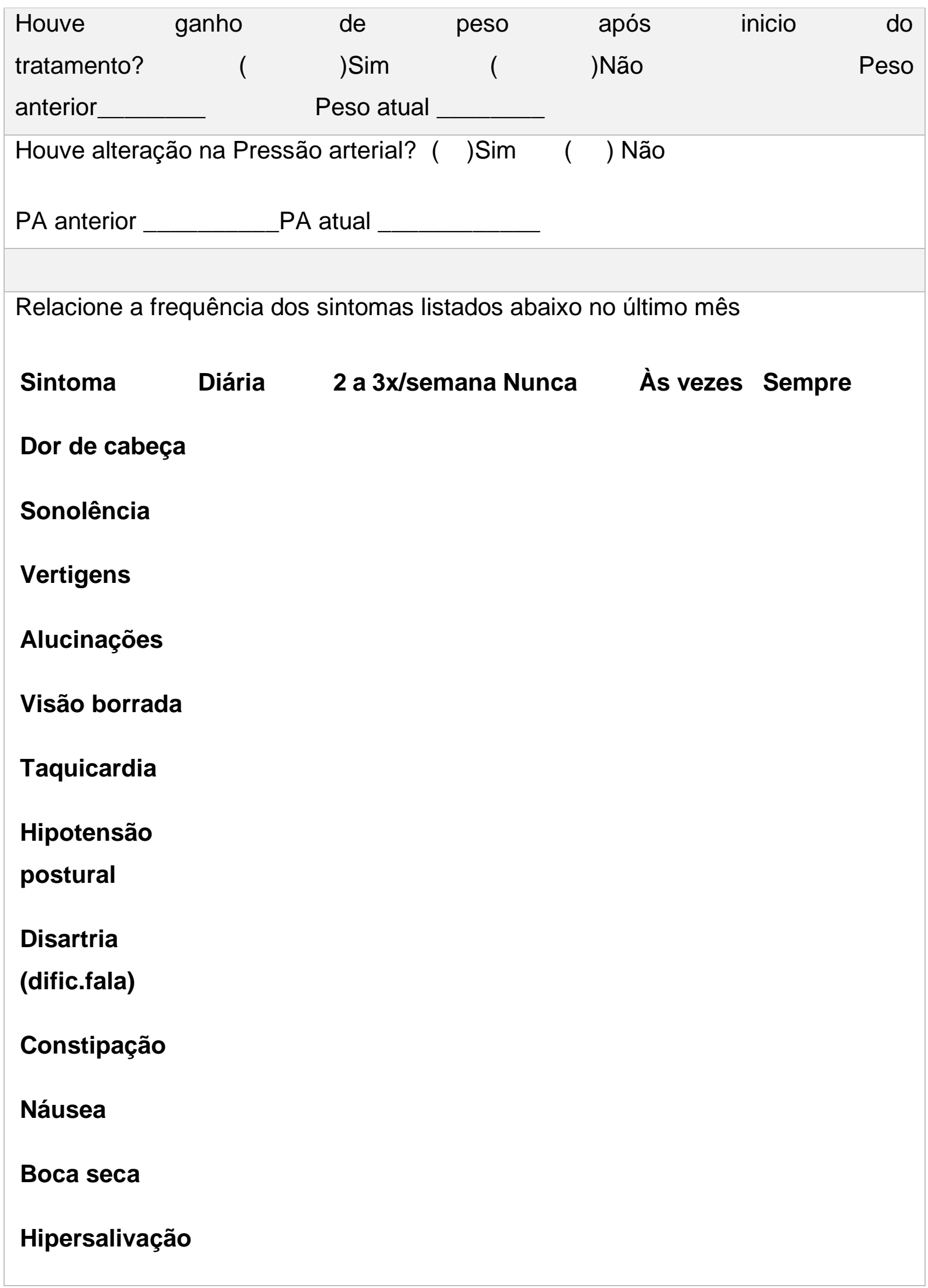




\section{Retenção \\ urinária \\ Incontinência \\ Urinaria}

Sudorese

Fadiga/

Cansaço

Responsável pelo preenchimento:

Conduta Farmacêutica:

Fonte: Elaboração própria

O formulário de seguimento farmacoterapêutico proposto visa aprimorar o tratamento e ser uma ferramenta rápida e possível de ser aplicada pelo paciente e familiares e/ou cuidadores sem conhecimento especifico da patologia.

\section{CONCLUSÃO}

A proposta de elaboração do formulário de seguimento farmacoterapêutico é identificar as reações adversas que possam influenciar a adesão ao tratamento farmacológico e reduzir os efeitos indesejados do tratamento como consequência melhorar a qualidade de vida paciente.

Para o paciente, a elaboração desse formulário significa uma possibilidade de um atendimento mais seguro e mais eficiente pelo farmacêutico, uma vez que permite o 
resgate do histórico dos acontecimentos vividos por ele em decorrência do uso do medicamento.

Os registros de informações existentes podem dispensar ou simplificar indagações e exames complementares, reduzindo internações, intervenções médicas e tempo de permanência nos serviços de saúde decorrente das reações adversas.

Em relação aos profissionais da área da saúde o registro dessas informações poderá contribuir para a integração da equipe de saúde, possibilitando a coordenação do cuidado. No ensino e na pesquisa, possibilita o conhecimento das reações adversas, histórico de medicamentos utilizados, limitações ou agravos à saúde promovendo maior conhecimento e intervenções sobre a temática.

No atendimento ao paciente, o farmacêutico, pode orientá-lo sobre o uso correto dos medicamentos prescritos, com intenção de melhorar os efeitos terapêuticos e reduzir a probabilidade de aparecimentos das reações adversas, interações medicamentosas e toxicidade.

Esse processo traz grandes benefícios e proporciona maior reconhecimento profissional ao farmacêutico fortalecendo o vínculo nas ações de saúde no acompanhamento de pacientes com esquizofrenia refratária.

\section{REFERÊNCIA}

BARKHOF, Emile et al. Interventions to improve adherence to antipsychotic medication in patients with schizophrenia-a review of the past decade. European Psychiatry, v. 27, n. 1, p. 9-18, 2012.

BATISTA, FPH et al. Prevalencia de síndrome metabólico en pacientes con esquizofrenia refractaria. Ciencia y enfermería, v. 22, n. 3, p. 11-24, 2016.

BENNETT LMD et al. Patients' preferences related to benefits, risks, and formulations of schizophrenia treatment. Psychiatric Services, v. 66, n. 7, p. 719-726, 2015. 
BRASIL. Ministério da Saúde. Portaria SAS/MS no 364, de 9 de abril de 2013. Protocolo clínico e diretrizes terapêuticas de esquizofrenia. Disponível em < http://portalarquivos.saude.gov.br/images/pdf/2014/abril/02/pcdt-esquizofrenialivro2013.pdf> Acesso 15.nov.18 ás16h.

BRASIL. Portal da saúde. Formulário padronizado para realização da consulta farmacêutica.

Disponível em

<http://portalsaude.saude.gov.br/images/docx/2015/abril/16/FORMUL--RIOPADRONIZADO-PARA-REALIZA----O-DA-CONSULTA-FARMAC--UTICAdisponibilizado-no-site.docx> Acesso 15.jan.19 ás 15h.

BRASIL. Conselho Federal de Farmácia. Modelo de formulário para documentação de serviços clínicos. Disponível em: http://www.cff.org.br/noticia. php?id=2581\&titulo=CFF+disponibilizamodelos+de+form ul\%C3\%A1rios+para+documenta\%C3\% A7\%C3\%A3o+de+servi\%C3\%A7os+cl\%C3\%ADnicos>Acesso 15.jan.19 ás 14h

CAMPANA, Mariana Campos; SOARES, Marcos Hirata. Familiares de pessoas com esquizofrenia: sentimentos e atitudes frente ao comportamento agressivo. Cogitare Enfermagem, v. 20, n. 2, 2015.

CEZARETTO, Marceli et al. Perfil clínico e sociodemográfico de pacientes com esquizofrenia refratária tratados em um centro terciário. Jornal Brasileiro de Psiquiatria, v. 63, n. 3, p. 185-190, 2014.

CLOZAPINA medicamento genérico. Jose Carlos Módolo. São Paulo.Cristalia. Disponível

em: $<$ http://www.anvisa.gov.br/datavisa/fila_bula/frmVisualizarBula.asp?pNuTransacao=1 175432015\&pldAnexo=2444983> Acesso em 15.nov.18 ás $20 \mathrm{~h}$.

FEGADOLLI, Claudia et al. Farmacêuticos integrando equipes de cuidado em saúde mental no Sistema Único de Saúde (SUS): uma reflexão coletiva na cidade de São Paulo. Interface-Comunicação, Saúde, Educação, v. 20, p. 1093-1098, 2016. 
FREITAS, Pedro Henrique Batista de et al. Esquizofrenia refratária: qualidade de vida e fatores associados. Acta Paulista de Enfermagem, v. 29, n. 1, p. 60-68, 2016.

FREITAS, PHB; NUNES, FDD; PINTO, JAF; et al. Síndrome metabólica em pacientes com esquizofrenia. Rev. enferm. Cent.-Oeste Min $_{1}$ 2016, 6(1):1976-1993. Disponível em: http://pesquisa.bvsalud.org/enfermagem/resource/pt/bde-28449> Acesso 10.dez.18 ás 20h.

GOLAN, DE; TASHJIAN, AH; ARMSTRONG, EJ; et al. Princípios de Farmacologia: a Base Fisiopatológica da Farmacoterapia, 2ed. Rio de Janeiro, Guanabara Koogan, 2009, 177-180.

KHAN, Afaque H.; ZAIDI, Samina. Clozapine: improvement of negative symptoms of schizophrenia. Cureus, v. 9, n. 12, 2017.

HANSEN, Nayara Fernanda et al. A sobrecarga de cuidadores de pacientes com esquizofrenia: uma revisão integrativa da literatura. Revista eletrônica de enfermagem, v. 16, n. 1, p. 220-7, 2014.

MAGALHÃES, Josiléia Félix et al. Estratégias de enfrentamento de mulheres cuidadoras de pessoas com esquizofrenia. Revista de Pesquisa: Cuidado é fundamental online, v. 10, n. 3, p. 793-800, 2018.

OLIVEIRA, DPA; LEITE, ESM; ARAUJO, GM, et al. Vivências e qualidade de vida de portadores de esquizofrenia. Journal of Medicine and Healt Promotion, 2017, 2(3):535-543. Disponível em http://jmhp.fiponline.edu.br/pdf/cliente=13de8b335b0a80db19c7acdd64fe02483c.pdf> Acesso 26.nov.18 ás 21h.

PEREIRA, ADU; MUSSEL, E; BARROS, HL, et al. Protocolos Clínicos e Diretrizes Clinicas, Esquizofrenia Refratária. Fundação Hospitalar do Estado de Minas Gerais, 2013, (30):101-130. Disponível em <http://www.fhemig.mg.gov.br/index.php/docman/Proto colos_Clinicos-1/126-030esquizofrenia-refrataria-07082014/files> Acesso:15.nov.18 ás 16h 
PORRAS-SEGOVIA, Alejandro et al. Rapid-onset clozapine-induced loss of glycaemic control: case report. BJPsych open, v. 3, n. 3, p. 138-140, 2017.

RANG, H.P; DALE, M.M; RITTER, J.M, et al. Farmacologia, 6ed. Rio de Janeiro, Elsevier, 2007, 551-553.

SILVA, Sarah Nascimento; LIMA, Marina Guimarães. Assistência Farmacêutica na Saúde Mental: um diagnóstico dos Centros de Atenção Psicossocial. Ciência \& Saúde Coletiva, v. 22, p. 2025-2036, 2017.

SILVA, Amanda Mendes et al. Esquizofrenia: Uma revisão bibliográfica. UNILUS Ensino e Pesquisa, v. 13, n. 30, p. 18-25, 2016.

SISKIND, Dan et al. Clozapine v. first-and second-generation antipsychotics in treatment-refractory schizophrenia: systematic review and meta-analysis. The British Journal of Psychiatry, v. 209, n. 5, p. 385-392, 2016.

WARNEZ, Stephanie; ALESSI-SEVERINI, Silvia. Clozapine: a review of clinical practice guidelines and prescribing trends. BMC psychiatry, v. 14, n. 1, p. 1-5, 2014.

WHO. Organização Mundial de Saúde. Esquizofrenia. Disponível em $<$ https://www.who.int/topics/schizophrenia/es/> Acesso 13.dez.18 às 20h40min. (2019)

Enviado: Agosto, 2020.

Aprovado: Setembro, 2020. 